journal club

\title{
Autismus beim Kind durch Antidepressiva?
}

Fragestellung: Erhöht eine antidepressive Medikation der Mutter das Risiko für Autismus beim Kind?

Hintergrund: In Europa nehmen 3-8\% der Schwangeren Antidepressiva ein. Diskutiert wird ein möglicher Zusammenhang zwischen Antidepressivaeinnahme und mentalen Entwicklungsauffälligkeiten des Kindes einschließlich Autismus.

Patienten und Methodik: Die Beobachtungsstudie schloss 254.610 Kinder und Jugendliche (vier bis 17 Jahre) ein. Um einen möglichen Zusammenhang fundierter beurteilen zu können, verfolgten die Autoren vier Auswertungsansätze. Neben einer Regressionsanalyse wurden unter Berücksichtigung mütterlicher Charakteristika mittels Propensity-Score exponierte und nicht exponierte Patientinnen im Verhältnis 1:4 „gematcht“. In einem diskordanten Geschwisteransatz wurden Familien untersucht, bei denen mindestens ein Kind die Diagnose Autismus hatte und mindestens ein weiteres Kind nicht und auch die vorgeburt-

Rai D, Lee BK, Dalman C et al. Antidepressants during pregnancy and autism in offspring: population based cohort study. BMJ 2017; 358: j2811 liche Antidepressivaexposition diskordant war. Schließlich wurde die Antidepressivaeinnahme des Vaters als Negativkontrolle untersucht.
Ergebnisse: Von 3.342 Kindern, deren Mütter Antidepressiva eingenommen hatten, wurde bei $136(4,1 \%)$ Autismus diagnostiziert, im Vergleich zu 353 Kindern (2,9\%) von 12.325 Müttern, die zwar eine psychiatrische Diagnose hatten, aber keine Antidepressiva eingenommen hatten. Die Signifikanz beruhte auf den Autismusfällen ohne intellektuelle Beeinträchtigung, jene mit intellektueller Einschränkung waren nicht signifikant häufiger. Bei den SSRI war die Rate an Autismus geringer (3,9\%) als bei anderen Antidepressiva (4,4\%). Von den 238.943 Kindern, deren Mütter weder Antidepressiva einnahmen, noch eine psychiatrische Diagnose aufwiesen, hatten 4.489 (2,1\%) Autismus. Auch beim Propensity-Score-Matching wurde eine signifikant höhere Rate von Autismus bei den Antidepressivaexponierten beobachtet. Beim diskordanten Geschwisteransatz ergab sich ebenfalls eine Assoziation, die allerdings nicht signifikant war. Die Antidepressivaeinnahme des Vaters ergab keine Assoziation. Die Autoren errechneten, dass sich bei Weglassen der Antidepressiva die Anzahl der Autismusfälle um 2\% verringern würde.

Schlussfolgerungen: Ein kausaler Zusammenhang zwischen Antidepressivaeinnahme in der Schwangerschaft und Autismus beim Kind ist nicht auszuschließen. Eine entscheidendere Rolle spielen aber möglicherweise die mütterliche Erkrankung sowie nicht erfasste genetische und andere familiäre Faktoren.

\section{- Kommentar von Christof Schaefer, Berlin}

\section{Kein Grund, eine notwendige antidepressive Therapie vorzuenthalten}

Die bisher publizierten Studien haben zusammengenommen keinen unabhängigen Effekt der mütterlichen Antidepressivaeinnahme auf die Entstehung von Autismus beim Kind belegt. Auch diese methodisch anspruchsvolle Studie lässt Fragen offen. Der Einfluss von Schwere und Verlauf der mütterlichen psychiatrischen Erkrankung konnte ebenso wenig berücksichtigt werden, wie die mütterliche Komedikation mit Antiepileptika, die nahezu doppelt so häufig wie in der Vergleichsgruppe vorkam. Die trotz Warnhinweisen für Frauen im gebärfähigen Alter immer noch verordnete Valproinsäure erhöht erwiesenermaßen das Risiko für Autismusspektrumstörungen. Kritisch anzumerken ist auch, dass das von den Autoren beobachtete vergleichsweise geringere Autismusrisiko bei den (heute allgemein bevorzugten) SSRI nicht prominenter dargestellt wird. Unklar bleiben auch die Kriterien der Autismusdiagnose. Diese hat in den letzten 30 Jahren drastisch zugenommen. Zu welchen Anteilen dies einer Zunahme der Symptomatik geschuldet ist oder der sich ändernden diagnostischen Zuordnung, sei dahingestellt. Lobenswert ist, dass die Autoren ausdrücklich die kritische Reflektion ihrer Ergebnisse anmahnen. Bei vielen Beobachtungsstudien zum Arzneimittelrisiko in der Schwangerschaft werden signifikante Ergebnisse unkritisch als Beleg für Kausalität gedeutet. Selbst wenn man von einem kausalen Zusammenhang zwischen Antidepressiva und Autismus ausgeht, sollte der
Hinweis der Autoren beachtet werden, dass bei Weglassen der Antidepressiva nur $2 \%$ weniger Autismusfälle zu erwarten sind. Andererseits darf man nicht vergessen, was eine unbehandelte schwere depressive Symptomatik für Mutter und Kind bedeutet, und welche Folgen es hat, wenn einer exponierten Mutter unkritisch die Autismushypothese präsentiert wird und diese nun ängstlich auf die Pathologie ihres Kindes wartet und/oder von Selbstvorwürfen geplagt wird.

Hinsichtlich Risiken in der Schwangerschaft sind SSRI die am besten untersuchte Arzneimittelgruppe. Bei Neueinstellung werden Sertralin und Citalopram als Antidepressiva der ersten Wahl empfohlen. Dabei sollte es bleiben, wenn eine pharmakologische antidepressive Behandlung notwendig ist.

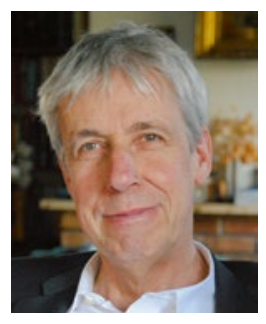

Prof. Dr. med. Christof Schaefer, Berlin

Leiter Pharmakovigilanz- und

Beratungszentrum für Embryonaltoxikologie, Charité - Universitätsmedizin Berlin E-Mail: christof.schaefer@charite.de 